\title{
Adjuvant vaginal cuff brachytherapy: dosimetric comparison of conventional versus 3-dimensional planning in endometrial cancer
}

\author{
Melis Gultekin, MD', Melek Tugce Yilmaz, MD', Fatih Biltekin, PhD', Deniz Yuce, MD, PhD², Sezin Yuce Sari, MD', \\ Fadil Akyol, MD', Ferah Yildiz, MD' \\ 'Department of Radiation Oncology, Faculty of Medicine, Hacettepe University, Ankara, Turkey, ${ }^{2}$ Department of Preventive Oncology, \\ Faculty of Medicine, Hacettepe University, Ankara, Turkey
}

\begin{abstract}
Purpose: To evaluate dosimetric differences between point-based 2-dimensional (2D) vaginal brachytherapy (VBT) treatment planning technique and volume-based 3-dimensional (3D) VBT method for endometrial cancer (EC).

Material and methods: Ten patients with uterine-confined EC treated with VBT were included in this study. All patients received $27.5 \mathrm{~Gy}$ in 5 fractions. Three different treatment plans were performed for each patient: plan A for dose prescribed to the entire vaginal wall thickness delineated via computed tomography guidance, plan B for dose prescribed to the vaginal mucosa/cylinder surface, and plan $\mathrm{C}$ for dose prescribed to $5 \mathrm{~mm}$ beyond the vaginal mucosa/cylinder surface. Dose-volume histograms (DVH) of treatment volumes and organs at risk (OARs) were evaluated and compared.

Results: DVH analysis of target volume doses $\left(\mathrm{D}_{100}, \mathrm{D}_{95}\right.$, and $\left.\mathrm{D}_{90}\right)$ showed a significant difference between plan $A$ and plan $\mathrm{B}(p=0.005)$, and plan $\mathrm{B}$ was found lower. $\mathrm{D}_{100}$ for plan $\mathrm{C}$ was significantly higher than plan $\mathrm{A}(p=0.009)$, but for $\mathrm{D}_{95}$ and $\mathrm{D}_{90}$, no statistically significant difference was found ( $p=0.028$ and $p=0.028$, respectively). In terms of OARs doses, including vagina, rectum, bladder, and sigmoid, $\mathrm{D}_{2 \mathrm{~cm}^{3}}$ doses were significantly higher in plan $\mathrm{A}$ than plan $\mathrm{B}$ $(p=0.009, p=0.009, p=0.005$, and $p=0.005$, respectively). All these doses were also significantly lower than in plan C ( $p=0.005, p=0.012$, and $p=0.013$, respectively), except for sigmoid ( $p=0.155)$.

Conclusions: In this dosimetric analysis, we have shown that the volume-based 3D VBT technique provides the ability to balance the target dose against the sparing of OARs. Therefore, in the new modern 3D treatment era, instead of normalization of the dose to standard reference points, customized 3D volume-based VBT planning should be recommended.

J Contemp Brachytherapy 2020; 12, 6: 601-605 DOI: https://doi.org/10.5114/jcb.2020.101694
\end{abstract}

Key words: endometrial cancer, brachytherapy, vaginal cuff brachytherapy, three-dimensional planning, dosimetric comparison

\section{Purpose}

Endometrial cancer (EC) is the most common gynecological cancer in post-menopausal women worldwide, and its incidence is increasing [1]. Primary treatment includes total hysterectomy $(\mathrm{TH})$ and bilateral salphingooopherectomy (BSO) with or without lymph node dissection. Adjuvant radiotherapy (RT) is generally reserved for patients with high recurrence risk factors. These risk factors include age $>60$ years, grade 3 histology, $\geq 50 \%$ myometrial invasion (MI), lymphovascular space invasion (LVSI), non-endometrioid tumor histology, lymph node metastases, and tumor extension into cervix or vagina $[2,3,4]$. Seventy percent of EC patients are diagnosed with International Federation of Gynecology and Obstetrics (FIGO) stage I disease, and are assigned into intermediate-risk (IR) or high-intermediate-risk (HIR) groups, when they are with either grade 3 histology or LVSI or $\geq 50 \%$ MI $[2,5]$. Several prospective trials showed that VBT produced similar oncological outcomes compared to external pelvic RT, but with significantly less toxicity in patients with uterine-confined disease $[6,7]$.

Traditionally, brachytherapy (BT) was performed using 2-dimensional (2D) point-based technique. However, based on the current development of computer science, 3-dimensional (3D) volume-based image-guided brachytherapy (IGBT) is the preferred technique, especially in patients with locally advanced cervical cancer [8]. 
With this 3D technique, target volume and organs at risk (OARs) are better defined when compared to 2D conventional BT, which leads to similar target coverage but significant reduction in OARs doses [9,10,11,12]. Even though there are several guidelines for IGBT planning in cervical cancer, recommendations for 3D vaginal BT (VBT) in terms of contouring, planning parameters, and dose constraints in EC are limited $[13,14,15,16]$. Recently, the American Brachytherapy Society (ABS) published a guideline for the use of adjuvant VBT after TH in patients with EC [15]. However, the treatment recommendations in this guideline mainly included the classical point-based approach of 2D era. In this study, we aimed to compare historical point-based 2D VBT treatment planning technique, in which the dose was prescribed to the vaginal surface or to the $5 \mathrm{~mm}$ deep from the vaginal mucosa with volume-based 3D VBT technique, where the dose was prescribed to the entire vaginal wall thickness, according to patients' anatomy.

\section{Material and methods}

\section{Patients}

In this study, 10 patients with uterine-confined EC who were treated between August 2018 and October 2018 at Hacettepe University, Department of Radiation Oncology were included. This study was approved by local institutional review board. The median age of the entire cohort was 60.5 years (range, 53-71 years). All patients were diagnosed with intermediate-risk disease, including stage IB, grade 1-2, endometrioid type EC without LVSI after surgery, and received adjuvant VBT only. A gynecological examination was done before the treatment to ensure that the patient's vaginal vault healing was completed. Radiotherapy was performed at least 4 weeks after surgery. All patients were treated with high-doserate (HDR) intracavitary brachytherapy, with a computed tomography (CT)-compatible single, central channel vaginal cylinder. During the gynecological examination, a suitable vaginal cylinder diameter (between 3-3.5 cm) was selected in accordance with the anatomy of patient's vagina. After the application of vaginal cylinder with a lubricant, patients underwent CT scan using Toshiba Aquilion LB CT Simulator (Toshiba Medical Systems, Otowara, Japan). All patients were requested to empty their bladder and rectum before CT scan. As a CT simulation protocol, $100-120 \mathrm{kVp}$ tube voltage, 300-350 mAs current value, and $2.5 \mathrm{~mm}$ slice thickness were used. After simulation processes, CT images were transferred to BrachyVision $^{\mathrm{TM}}$ treatment planning system (TPS) version 8.9 (Varian Medical Systems, Palo Alto, CA, USA) via digital imaging and communication in medicine (DICOM) connection.

\section{Treatment planning}

For the 3D VBT, proximal $3.5 \mathrm{~cm}$ of the vagina including the entire thickness of vaginal wall was contoured as clinical target volume (CTV) by a single physician. Bladder, rectum, sigmoid colon, small bowel, and the remaining vagina were also delineated as OARs $[17,18]$. A dose of $27.5 \mathrm{~Gy}$ in 5 fractions was prescribed. The goal of $3 \mathrm{D}$ treatment planning was that $95 \%$ of the CTV received at least $95 \%$ of the prescribed dose, and $100 \%$ of the CTV received at least $90 \%$ of the prescribed dose (plan A). In plan A, dose shaper tool and dwell control window available in BrachyVision ${ }^{\mathrm{TM}}$ TPS were used to obtain an optimum dose distribution, and volume prescription was performed to achieve similar dose distribution for all cases. Additionally, two different point-based treatment plans were performed with the dose specified at either vaginal mucosa/cylinder surface (plan B) or $5 \mathrm{~mm}$ beyond (plan C), according to the ABS recommendations (2D VBT). Geometrical optimization tool was used to prescribe the dose to the reference points for defined target length for each patient (Figure 1) [15]. For dosimetric comparison of treatment plans, median, mini-

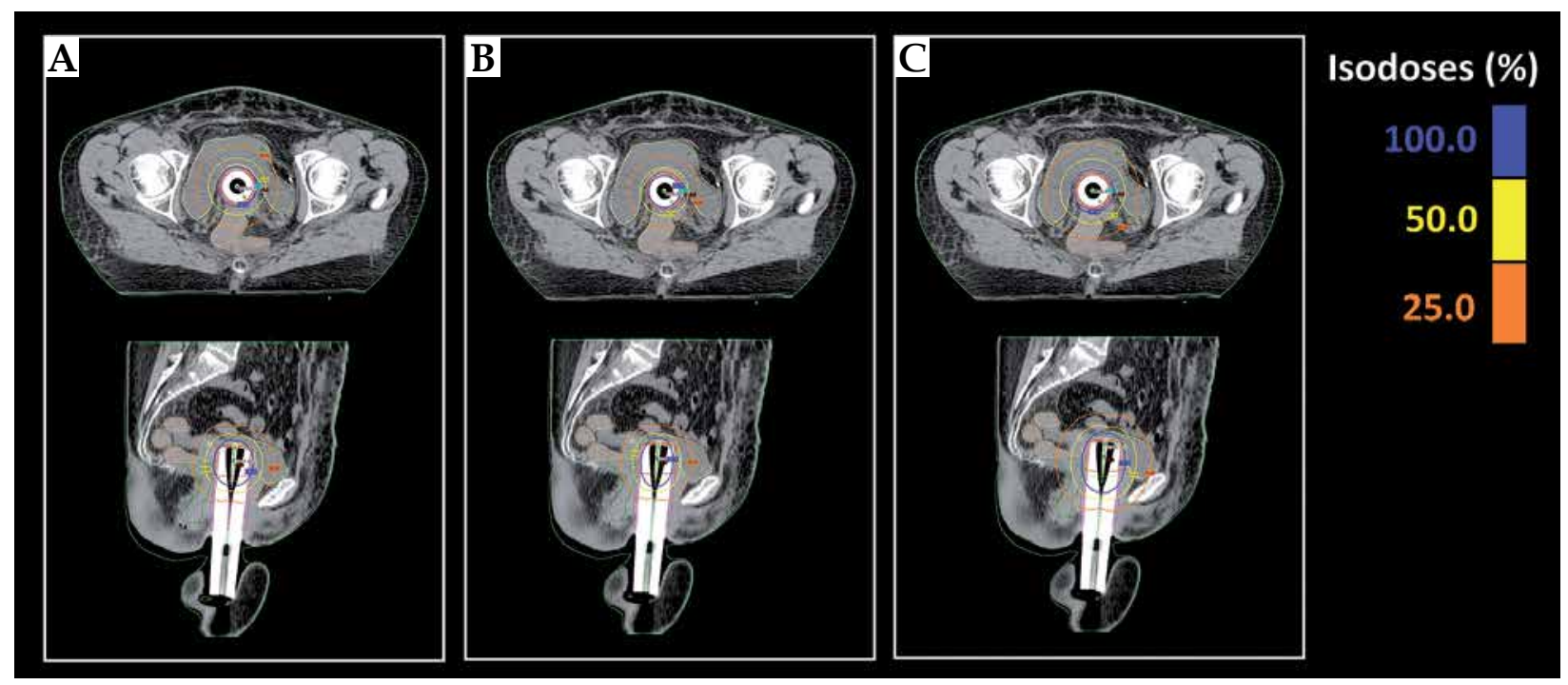

Fig. 1. Computed tomography images of different treatment plans with isodoses. A) Axial and sagittal sections for plan A, B) axial and sagittal sections for plan B, C) axial and sagittal sections for plan C 
mum, and maximum doses received by $100 \%$ of the CTV $\left(D_{100}\right), 95 \%$ of the CTV $\left(D_{95}\right)$, and $90 \%$ of the CTV $\left(D_{90}\right)$ and the minimum dose received in most exposed $2 \mathrm{~cm}^{3}$ of OARs $\left(\mathrm{D}_{2 \mathrm{~cm}^{3}}\right)$ were noted. HDR VBT was performed with ${ }^{192}$ Ir using GammaMed Plus iX brachytherapy unit (Varian Medical Systems, Palo Alto, CA, USA).

\section{Statistical analyses}

Descriptive statistics were presented using median, minimum, and maximum values. Three different VBT plans were compared using Friedman's non-parametric analysis of variance test, and Wilcoxon test was used for post-hoc pairwise comparisons with Bonferroni correction. All the reported $p$ values were two-sided, and a $p$-value of $<0.05$ was considered statistically significant. Statistical analyses were performed using SPSS 23.0 software (IBM Inc., Armonk, NY, USA).

\section{Results}

\section{Treatment volumes}

The median, minimum, and maximum values of $\mathrm{D}_{100}, \mathrm{D}_{95}$, and $\mathrm{D}_{90}$ of CTV are shown in Table 1. The median $\mathrm{D}_{100}$ was 439.5 cGy (range, 248-457 cGy) in plan A, 312.5 cGy (range, 180-355 cGy) in plan B, and 484.5 cGy (range, 250-529 cGy) in plan C. The median $\mathrm{D}_{95}$ was 553 cGy (range, 550-559 cGy) in plan A, 398.5 cGy (range, 353-450 cGy) in plan B, and 594.5 cGy in plan C (range, 530-663 cGy). Finally, the median $\mathrm{D}_{90}$ was $595 \mathrm{cGy}$ (range, 591-649cGy) in plan A, 429 cGy (range, 393-489cGy) in plan B, and 640.5 cGy in plan C (range, 586-720 cGy).

The comparisons of the target volumes between plans revealed that $\mathrm{D}_{100}(p<0.01), \mathrm{D}_{95}(p<0.01)$, and $\mathrm{D}_{90}$ $(p<0.01)$ were all significantly different between plans $\mathrm{A}$, $\mathrm{B}$, and $\mathrm{C}$. When the plan $\mathrm{A}$ was considered as a reference category, the values of target volumes were significantly lower in plan B $\left(p=0.005\right.$ for $\mathrm{D}_{100}, p=0.005$ for $\mathrm{D}_{95}$, and $p=0.007$ for $\mathrm{D}_{90}$ ).

In plan $C$, the target volume doses were statistically significantly higher for $D_{100}$ than plan $A(p=0.009)$, whereas for $D_{95}$ and $D_{90}$, there was a trend toward statistical significance ( $p=0.028$ and $p=0.028$, respectively).

In addition, since dose normalization was performed at a constant depth in plans $B$ and $C$, the $D_{95}$ values of 10 patients ranged from $353 \mathrm{cGy}$ to $450 \mathrm{cGy}$ in plan $\mathrm{B}$, and 530 cGy to 663 cGy in plan C on a wide scale. However, in plan $A$, the $D_{95}$ values varied in the range of 550-559 cGy, and $D_{95}$ values for CTVs were found equal to or greater than 550 cGy in all patients.

\section{Organs at risk}

Dose details received by $2 \mathrm{~cm}^{3}$ of the OARs are shown in Table 2. In plan $A$, vaginal $D_{2 \mathrm{~cm}^{3}}$ values were significantly higher compared to plan $\mathrm{B}(p=0.009)$, but significantly lower than plan $C(p=0.005)$. Again, $\mathrm{D}_{2 \mathrm{~cm}^{3}}$ values of the rectum were significantly higher in plan A compared to plan B ( $p=0.009)$, but there were significantly lower than in plan C $(p=0.012)$. Similarly, bladder $D_{2 \mathrm{~cm}^{3}}$ values were significantly higher in plan $\mathrm{A}$ than plan $\mathrm{B}$ $(p=0.005)$, but these doses were significantly lower than in plan $\mathrm{C}(p=0.013)$. Lastly, in plan $\mathrm{A}$, sigmoid $\mathrm{D}_{2 \mathrm{~cm}^{3}}$ values were significantly higher than plan $\mathrm{B}(p=0.005)$. There was no statistically significant difference for doses received by sigmoid between plan A and plan C $(p=0.155)$.

\section{Discussion}

Patients with stage I EC have a 5-year overall survival rates of more than $90 \%$ [19]. Due to long-term survival

Table 1. Median, minimum, and maximum values of the CTVs $\left(D_{100}, D_{95}\right.$, and $\left.D_{90}\right)$

\begin{tabular}{ccccc} 
Parameter & Median & Minimum & Maximum & $P$-value* \\
\hline $\mathrm{D}_{100}$ (cGy) & & & & $<0.01$ \\
\hline Plan A & 439.5 & 248 & 457 & \\
\hline Plan B & 312.5 & 180 & 355 & 0.005 \\
\hline Plan C & 484.5 & 250 & 529 & 0.009 \\
\hline$D_{95}$ (cGy) & & & & $<0.01$ \\
\hline Plan A & 553 & 550 & 559 & \\
\hline Plan B & 398.5 & 353 & 450 & 0.005 \\
\hline Plan C & 594.5 & 530 & 663 & 0.028 \\
\hline$D_{90}$ (cGy) & & & & $<0.01$ \\
\hline Plan A & 595 & 591 & 649 & \\
\hline Plan B & 429 & 393 & 489 & 0.007 \\
\hline Plan C & 640.5 & 586 & 720 & 0.028
\end{tabular}

${ }^{*} P$-value of Friedman's statistics for $D_{100}, D_{95}$, and $D_{90}$, post-hoc $p$-values of plan $B$ and $C$ comparisons with reference plan $A$

Table 2. The dose received by $2 \mathrm{~cm}^{3}$ of the vagina, rectum, bladder, and sigmoid colon

\begin{tabular}{lcccc} 
Parameter & Median & $\begin{array}{c}\text { Mini- } \\
\text { mum }\end{array}$ & $\begin{array}{c}\text { Maxi- } \\
\text { mum }\end{array}$ & P-value* \\
\hline Vaginal 2cm $^{3}$ (cGy) & & & & 0.002 \\
\hline Plan A & 369 & 324 & 458 & \\
\hline Plan B & 320.5 & 260 & 350 & 0.009 \\
\hline Plan C & 519.5 & 411 & 714 & 0.005 \\
\hline Rectal D $\mathrm{cm}^{3}$ (cGy) & & & & 0.001 \\
\hline Plan A & 374.5 & 230 & 483 & \\
\hline Plan B & 293 & 216 & 455 & 0.009 \\
\hline Plan C & 437 & 330 & 519 & 0.012 \\
\hline Bladder D $2 \mathrm{~cm}^{3}(\mathrm{cGy})$ & & & & $<0.01$ \\
\hline Plan A & 467.5 & 391 & 543 & \\
\hline Plan B & 344.5 & 299 & 406 & 0.005 \\
\hline Plan C & 519.5 & 449 & 555 & 0.013 \\
\hline Sigmoid colon $\mathrm{D}_{2 \mathrm{~cm}^{3}}(\mathrm{cGy})$ & & & & 0.001 \\
\hline Plan A & 199 & 48 & 277 & \\
\hline Plan B & 145 & 31 & 216 & 0.005 \\
\hline Plan C & 197.5 & 48 & 232 & 0.155
\end{tabular}

${ }^{*}$-value of Friedman's statistics for vaginal $D_{2 \mathrm{~cm}^{3}}$, rectal $D_{2 \mathrm{~cm}^{3}}$, bladder $D_{2 \mathrm{~cm}^{3}}$, sigmoid colon $D_{2 \mathrm{~cm}^{3}}$, post-hoc p-values of plan $B$ and $C$ comparisons with reference plan $A$ 
rates, emphasis should be placed on minimizing treatment-related side effects that may compromise patients' quality of life (QOL). In external pelvic RT, QOL analyzed in post-operative radiation therapy in endometrial carcinoma (PORTEC)- 1 trial have shown that $20 \%$ of patients suffer from late gastrointestinal (GI) and genitourinary (GU) side effects [20,21]. Compared to external pelvic RT, VBT allows for higher doses to the vaginal mucosa, while providing greater protection for surrounding OARs. In PORTEC-2 trial, VBT alone had been demonstrated as effective as external pelvic RT in preventing vaginal recurrence without survival rate difference, and these patients with VBT alone had superior outcomes, especially regarding diarrhea, fecal incontinence, and social functioning $[7,22]$. However, in this study, despite the reduction of GI toxicity, grade 1-3 vaginal toxicity increased in the VBT arm compared to the external pelvic RT arm ( $36.6 \%$ vs. $17.7 \%, p<0.05)$. Although it is less common than external pelvic RT, severe toxicity rates were reported to be in a range of $0-5.2 \%$ with VBT, which included vaginal stenosis, vaginal necrosis, rectovaginal fistula, and urethral stricture [23]. In this respect, volume-based 3D VBT treatment planning can provide an advantage by decreasing the OARs doses when comparing historical point-based 2D VBT, and can minimize higher doses in the vagina, especially in parts with a low-risk for disease recurrence. Additionally, target volume can be contoured 3-dimensionally, and customized treatment plans can be prepared in accordance with patients' anatomy. Although this advantage was demonstrated in cervical cancer IGBT, there is no clear data on this issue in EC [24,25].

Currently, it is a common practice to prescribe a dose to a depth of $5 \mathrm{~mm}$ from the mucosal surface, which was first recommended by Sorbe and Smeds [26]. This recommendation was based on the idea that vaginal lymphatics would lie in the first $5 \mathrm{~mm}$ [26]. In another study performed by Choo et al., specimens of full-thickness vaginal biopsies of 13 patients revealed that $95 \%$ of vaginal lymphatics were located in the first $3 \mathrm{~mm}$ [27]. The ABS recommends prescribing the dose to the applicator surface or depth of $5 \mathrm{~mm}$ according to clinical protocols [15]. However, treatment plans according to these recommendations may lead to higher OARs doses, when the vaginal mucosa is thin (atrophic), or may lead to lower target volume coverage in obese patients, resulting an unfavorable therapeutic index. In a retrospective study performed by Onsrud et al., adjuvant VBT was performed in 217 patients with stage I-II EC [13]. They compared late reactions of patients receiving treatment to a standard depth of $5 \mathrm{~mm}$ with treatment depths 3,4 , and $5 \mathrm{~mm}$ according to the vaginal mucosal thickness on gynecological examination. They found that the GI, GU, and vaginal side effects were significantly decreased with the individualized treatment plans compared to the standard treatment.

In this present dosimetric analysis, we compared the point-based 2D VBT treatment planning technique to volume-based 3D VBT procedure in patients with intermediate-risk EC. In the point-based technique, the dose was prescribed historically to the vaginal mucosa (or applicator surface) or $5 \mathrm{~mm}$ beyond, while in the vol- ume-based technique, the entire vaginal wall thickness was contoured with image guidance and the dose was prescribed to this volume considering the patient's anatomy. We have achieved statistically significant dosimetric advantage by 3D VBT (plan A) technique compared to 2D VBT technique, in which the dose was prescribed at the $5 \mathrm{~mm}$ depth from the vaginal mucosa (plan C) in terms of rectum and bladder $\mathrm{D}_{2 \mathrm{~cm}}$ doses. Also, we obtained better dose coverage in the CTV with 3D VBT compared to $2 \mathrm{D}$ technique. The reason why there are sparse consensus recommendations for 3D VBT in EC is that OARs' doses are quite low in patients treated with adjuvant VBT alone. However, when there is a need for using both external pelvic RT and VBT due to surgical margin positivity or stage II disease or extensive LVSI, OARs' doses may exceed tolerance limits and 3D VBT can provide an advantage, especially in these cases. It seems clear that in the 3D treatment era, it is necessary to determine the thickness of vaginal wall according to CT data, which provides dose optimization based on proximity to OARs.

In the literature, there are no randomized studies regarding the optimal dose, fractionation, or prescription point for VBT in EC. Many different fractionation schemes were applied for adjuvant VBT [28]. VBT dose depends on the dose specification point, length of vagina treated, and whether EBRT is applied. One of the most frequently used schemes for patients treated with VBT alone according to the ABS recommendations is $5 \times 6 \mathrm{~Gy}$, if the dose is prescribed to the vaginal mucosa, and $5 \times 5$ Gy if the dose is prescribed to the $5 \mathrm{~mm}$ beyond the vaginal mucosa [15]. In our VBT alone protocol, a total of 27.5 Gy was applied in 5 fractions. We found that 3D VBT planning yields better dose coverage compared to 2D VBT. Due to the dose normalization at a standard point, $\mathrm{D}_{95}$ values of 10 patients varied in a wide scale between 350 cGy and 450 cGy for plan B and 530 cGy and 663 cGy for plan C. However, in plan A, these values ranged from 550 cGy to 559 cGy and were equal or greater than 550 cGy in all patients. When the dose was prescribed to the vaginal mucosa (plan B) in 2D VBT planning, target coverage was inadequate. According to the ABS recommendations, if the dose is prescribed to the vaginal mucosa, the fraction dose should be increased [29].

At present, the use of CT imaging is gradually increasing in VBT planning in EC. A recent survey of the ABS members indicated that for adjuvant VBT, $64 \%$ of the respondents utilized CT imaging for VBT planning [30]. Even though the majority of respondents preferred CT-based treatment planning in VBT, they still used dose prescription to $0.5 \mathrm{~cm}$ depth or mucosal surface similar to $2 \mathrm{D}$ VBT technique, with point-dose calculation. We believe that it is more sensible to perform the treatment planning volumetrically rather than point-dose calculation, which would lead to a decrease of OARs' doses and hot points in the low-risk part of vagina.

\section{Conclusions}

As a result, a normalization of the dose to standard reference points when planning VBT in patients with EC brings uncertainties in terms of CTV and OARs doses. 
In these cases, a customized 3D volume-based VBT planning, as in cervical cancers, can minimize these uncertainties. With this approach, the target volume is covered sufficiently, and unnecessary irradiation of OARs can be prevented. Therefore, it may be useful to update dose optimization and normalization recommendations for VBT according to the new modern 3D treatment era.

\section{Disclosure}

The authors report no conflict of interest.

\section{References}

1. Siegel RL, Miller KD, Jemal A. Cancer statistics, 2020. CA Cancer I Clin 2020; 70: 7-30.

2. Colombo N, Creutzberg C, Amant $\mathrm{F}$ et al. ESMO-ESGOESTRO Consensus Conference on Endometrial Cancer: diagnosis, treatment and follow-up. Ann Oncol 2016; 27: 16-41.

3. Klopp A, Smith BD, Alektiar K et al. The role of postoperative radiation therapy for endometrial cancer: Executive summary of an American Society for Radiation Oncology evidence-based guideline. Pract Radiat Oncol 2014; 4: 137-144.

4. Meyer LA, Bohlke K, Powell MA et al. Postoperative Radiation Therapy for Endometrial Cancer: American Society of Clinical Oncology Clinical Practice Guideline Endorsement of the American Society for Radiation Oncology Evidence-Based Guideline. J Clin Oncol 2015; 33: 2908-2913.

5. Pecorelli S. Revised FIGO staging for carcinoma of the vulva, cervix, and endometrium. Int J Gynaecol Obstet 2009; 105 103-104.

6. Aalders JG, Abeler V, Kolstad P. Recurrent adenocarcinoma of the endometrium: a clinical and histopathological study of 379 patients. Gynecol Oncol 1984; 17: 85-103.

7. Nout RA, Smit VT, Putter H et al. Vaginal brachytherapy versus pelvic external beam radiotherapy for patients with endometrial cancer of high-intermediate risk (PORTEC-2): an open-label, non-inferiority, randomised trial. Lancet 2010; 375: 816-823.

8. Haie-Meder C, Potter R, Van Limbergen E et al. Recommendations from Gynaecological (GYN) GEC-ESTRO Working Group (I): concepts and terms in 3D image based 3D treatment planning in cervix cancer brachytherapy with emphasis on MRI assessment of GTV and CTV. Radiother Oncol 2005; 74: 235-245.

9. Pötter R, Georg P, Dimopoulos JC et al. Clinical outcome of protocol based image (MRI) guided adaptive brachytherapy combined with 3D conformal radiotherapy with or without chemotherapy in patients with locally advanced cervical cancer. Radiother Oncol 2011; 100: 116-123.

10. Ribeiro I, Janssen H, De Brabandere $\mathrm{M}$ et al. Long term experience with 3D image guided brachytherapy and clinical outcome in cervical cancer patients. Radiother Oncol 2016; 120: 447-454.

11. Sturdza A, Potter R, Fokdal LU et al. Image guided brachytherapy in locally advanced cervical cancer: Improved pelvic control and survival in RetroEMBRACE, a multicenter cohort study. Radiother Oncol 2016; 120: 428-433.

12. Tanderup K, Fokdal LU, Sturdza A et al. Effect of tumor dose, volume and overall treatment time on local control after radiochemotherapy including MRI guided brachytherapy of locally advanced cervical cancer. Radiother Oncol 2016; 120: 441-446.

13. Onsrud M, Strickert T, Marthinsen AB. Late reactions after postoperative high-dose-rate intravaginal brachytherapy for endometrial cancer: a comparison of standardized and indi- vidualized target volumes. Int J Radiat Oncol Biol Phys 2001; 49: 749-755.

14. Small W, Jr. To plan or not to plan? That is the question. Brachytherapy 2006; 5: 216-217.

15. Small W, Jr., Beriwal S, Demanes DJ et al. American Brachytherapy Society consensus guidelines for adjuvant vaginal cuff brachytherapy after hysterectomy. Brachytherapy 2012; 11: 58-67.

16. Miller DA, Richardson S, Grigsby PW. A new method of anatomically conformal vaginal cuff HDR brachytherapy. Gynecol Oncol 2010; 116: 413-418.

17. Gay HA, Barthold HJ, O'Meara E et al. Pelvic normal tissue contouring guidelines for radiation therapy: a Radiation Therapy Oncology Group consensus panel atlas. Int J Radiat Oncol Biol Phys 2012; 83: e353-362.

18. Westerveld H, Potter R, Berger D et al. Vaginal dose point reporting in cervical cancer patients treated with combined 2D/3D external beam radiotherapy and 2D/3D brachytherapy. Radiother Oncol 2013; 107: 99-105.

19. Creasman WT, Odicino F, Maisonneuve P et al. Carcinoma of the corpus uteri. Int J Gynaecol Obstet 2006; 95 Suppl 1: S105-S143.

20. Creutzberg CL, Nout RA, Lybeert ML et al. Fifteen-year radiotherapy outcomes of the randomized PORTEC-1 trial for endometrial carcinoma. Int J Radiat Oncol Biol Phys 2011; 81: e631-638.

21. Nout RA, van de Poll-Franse LV, Lybeert ML et al. Longterm outcome and quality of life of patients with endometrial carcinoma treated with or without pelvic radiotherapy in the post operative radiation therapy in endometrial carcinoma 1 (PORTEC-1) trial. J Clin Oncol 2011; 29: 1692-1700.

22. Nout RA, Putter H, Jurgenliemk-Schulz IM et al. Five-year quality of life of endometrial cancer patients treated in the randomised Post Operative Radiation Therapy in Endometrial Cancer (PORTEC-2) trial and comparison with norm data. Eur J Cancer 2012; 48: 1638-1648.

23. Harkenrider MM, Block AM, Alektiar KM et al. American Brachytherapy Task Group Report: Adjuvant vaginal brachytherapy for early-stage endometrial cancer: A comprehensive review. Brachytherapy 2017; 16: 95-108.

24. Bahadur YA, Hassouna AH, Constantinescu CT et al. Three-dimension anatomy-based planning optimization for high dose rate vaginal vault brachytherapy. Saudi Med J 2012; 33: 640-647.

25. Beriwal S, Kim H, Heron DE, Selvaraj R. Comparison of $2 \mathrm{D}$ vs. 3D dosimetry for Rotte ' $\mathrm{Y}$ ' applicator high dose rate brachytherapy for medically inoperable endometrial cancer. Technol Cancer Res Treat 2006; 5: 521-527.

26. Sorbe BG, Smeds AC. Postoperative vaginal irradiation with high dose rate afterloading technique in endometrial carcinoma stage I. Int J Radiat Oncol Biol Phys 1990; 18: 305-314.

27. Choo JJ, Scudiere J, Bitterman P et al. Vaginal lymphatic channel location and its implication for intracavitary brachytherapy radiation treatment. Brachytherapy 2005; 4: 236-240.

28. Harkenrider MM, Grover S, Erickson BA et al. Vaginal brachytherapy for postoperative endometrial cancer: 2014 Survey of the American Brachytherapy Society. Brachytherapy 2016; 15: 23-29.

29. Nag S, Erickson B, Parikh S et al. The American Brachytherapy Society recommendations for high-dose-rate brachytherapy for carcinoma of the endometrium. Int J Radiat Oncol Biol Phys 2000; 48: 779-790.

30. Martell K, Doll C, Barnes EA et al. Radiotherapy practices in postoperative endometrial cancer: A survey of the ABS membership. Brachytherapy 2019; 18: 741-746. 